\title{
Positive curvature and rational ellipticity
}

\author{
MANUEL AMANN \\ LEE KENNARD
}

\begin{abstract}
Simply connected manifolds of positive sectional curvature are speculated to have a rigid topological structure. In particular, they are conjectured to be rationally elliptic, ie to have only finitely many non-zero rational homotopy groups. In this article, we combine positive curvature with rational ellipticity to obtain several topological properties of the underlying manifold. These results include an upper bound on the Euler characteristic and new evidence for a couple of well-known conjectures due to Hopf and Halperin. We also prove a conjecture of Wilhelm for even-dimensional manifolds whose rational type is one of the known examples of positive curvature.
\end{abstract}

53C20; 57N65, 55P62

\section{Introduction}

The question of whether a manifold admits a positively curved Riemannian metric has been addressed in various ways. Surprisingly, all this effort did not bring forth a long list of examples. All the known simply connected examples have one striking topological property in common. They are what are called rationally elliptic spaces, meaning that their total rational homotopy $\pi_{*}(\cdot) \otimes \mathbb{Q}$ is finite-dimensional. (See Ziller [50] for a survey of positively and non-negatively curved examples, see Dearricott [10], Grove, Verdiani and Ziller [20], and Petersen and Wilhelm [34] for new examples in dimension seven, and see Felix, Halperin and Thomas [12] for a reference on rational homotopy theory.)

Rational ellipticity on its own has interesting consequences. In particular, it implies the upper bound on the sum of the Betti numbers conjectured by Gromov for non-negatively curved manifolds.

A conjecture of Bott, Grove and Halperin states that a non-negatively curved manifold is rationally elliptic (see Grove [16, Section 5]). The Bott-Grove-Halperin conjecture holds for homogeneous spaces (in fact, for biquotients), and it is known for manifolds of cohomogeneity one and two (see Grove and Halperin [18] and Yeager [47]). In this article, we consider a number of conjectures for positively curved manifolds in the special case where the manifold is rationally elliptic. 
Our first result provides new evidence for a conjecture of Wilhelm. The conjecture states that if $M \rightarrow B$ is a Riemannian submersion of manifolds with positive sectional curvature, then $\operatorname{dim} M<2 \operatorname{dim} B$. If $M$ is not compact, Walschap [42] proved that there are no such non-trivial submersions. In the compact case, $M \rightarrow B$ is a fibration, so the conclusion is equivalent to the condition $\operatorname{dim} F<\operatorname{dim} B$, where $F$ is the fiber.

Theorem A Let $F \hookrightarrow M^{n} \rightarrow B$ be a non-trivial fibration of simply connected, compact manifolds. Suppose $M$ satisfies one of the following:

- $H^{*}(M ; \mathbb{Q})$ is singly generated as an algebra.

- $M$ is rationally equivalent to a Wallach flag manifold $W^{6}=\mathrm{SU}(3) / T^{2}, W^{12}=$ $\mathrm{Sp}(3) / \mathrm{Sp}(1)^{3}$, or $W^{24}=\mathrm{F}_{4} / \mathrm{Spin}(8)$.

- $\quad M$ is rationally equivalent to a hermitian symmetric space, and $M$ admits a metric with positive sectional curvature and an effective, isometric torus action of rank at least $2 \log _{2} n+6$.

Then it holds that

$\operatorname{dim} F<\operatorname{dim} B$,

except for the last case in which equality $\operatorname{dim} F=\operatorname{dim} B$ might hold if $n=4 m$ and $M$ is rationally equivalent to $\mathrm{SO}(2 m+2) / \mathrm{SO}(2 m) \times \mathrm{SO}(2)$.

The even-dimensional, simply connected, closed manifolds known to admit positive curvature are spheres, projective spaces, the Wallach flag manifolds, and the Eschenburg biquotient $\mathrm{SU}(3) / / T^{2}$. The last of these is rationally equivalent to the Wallach flag $\mathrm{SU}(3) / T^{2}$, so Theorem A confirms the Wilhelm conjecture for all of these rational types. Note that not all smooth manifolds with singly generated cohomology ring are spheres or projective spaces (see $\mathrm{Su}$ [38] for an example in dimension 32). Also note that, in the last item in the theorem, we do not require that there is a positively curved hermitian metric on $M$.

Theorem A is proved in Theorems 4.13, 4.17, 4.19. Compare Browder [8, Theorem 1, paper 202] for a similar result on spheres. In Corollary 4.14, we generalize the first item as follows: if $H^{*}(M ; \mathbb{Q})$ is freely generated as an algebra by two odd-degree generators of equal degree, then the fibration is trivial or $\operatorname{dim} F \leq \operatorname{dim} B$.

We remark that the entire theorem only uses rational structure and thus holds for rational fibrations of spaces which share the depicted rational homotopy types.

For the rest of our results we assume, in addition, a large degree of symmetry. In the 1990s, Grove propagated the idea of focusing on positively curved metrics that admit 
large isometry groups. The measure of symmetry we will consider in this paper is the symmetry rank, which is the rank of the isometry group. In other words the symmetry rank of a Riemannian manifold is at least $r$ if and only if there exists an effective, isometric action of a torus of dimension $r$.

Many topological classification results of varying strengths (diffeomorphism, homeomorphism, homotopy, etc) have been proven under the assumption that the symmetry rank is sufficiently large. The classification theorems of Grove and Searle [19] and Wilking [44] are prototypical examples. For related results and context we refer the reader to the surveys of Grove [17], Wilking [46] and Ziller [51].

We focus here on a much weaker topological classification problem, which is motivated by a conjecture of Hopf. The conjecture is that a compact, even-dimensional manifold with positive sectional curvature has positive Euler characteristic. Under the additional assumption of torus symmetry, there are many results that verify the conjecture (see Püttmann and Searle [35] and Rong and Su [36]). In Kennard [24] the conjecture was verified under the assumption that $n=\operatorname{dim}(M)$ is divisible by four and that the symmetry rank $r \geq 2 \log _{2} n-2$. In Amann and Kennard [3], the conjecture was established in all even dimensions under the assumptions that the symmetry rank $r \geq \log _{4 / 3} n$ and that the second, third, or fourth Betti number of $M$ vanishes. Here we prove the following:

Theorem B Let $M^{2 n}$ be a simply connected rationally elliptic manifold admitting a metric of positive curvature with symmetry rank at least $\log _{4 / 3}(2 n)$. Then the Euler characteristic of $M$ satisfies $\chi(M) \geq 2$.

Finally, we discuss a conjecture of Halperin. By a combination of the above conjectures of Bott-Grove-Halperin and Hopf, one would conclude that an even-dimensional, positively curved compact manifold is an $F_{0}$-space, ie is a rationally elliptic space with positive Euler characteristic. For the class of $F_{0}$-spaces, Halperin conjectured the following: For any fibration $F \hookrightarrow E \rightarrow B$ of simply connected spaces, if $F$ is $F_{0}$, then $H^{*}(E ; \mathbb{Q}) \cong H^{*}(B ; \mathbb{Q}) \otimes H^{*}(F ; \mathbb{Q})$ as $H^{*}(B ; \mathbb{Q})$-modules. In light of the conjectures of Bott-Grove-Halperin and Hopf, it is natural to consider Halperin's conjecture for even-dimensional positively curved manifolds. We prove the following in the presence of large symmetry rank.

Corollary C If $M^{n}$ is a rationally elliptic closed manifold with positive sectional curvature and symmetry rank $r \geq n / 8+2 \log _{2} n$, then $M$ satisfies the Halperin conjecture. 
We remark that by a result of Wilking [44, Theorem 5] (see also [3, Lemma 4.2, page 16]), one sees directly that the same result holds if $r \geq n / 6+2 \log _{2} n-3$. Indeed, Wilking's result implies that the rational cohomology algebra is generated by at most two elements, and the Halperin conjecture is known to hold for such spaces (see Theorem 1.2).

The main tool used to prove this result is the following theorem, which restricts the number of generators of the rational cohomology algebra. The theorem also improves on $[3$, Theorem $\mathrm{A}]$ in the special case of rationally elliptic manifolds.

Theorem D Let $M^{n}$ be rationally elliptic of positive curvature and with symmetry rank $r \geq n /(2 k)+2 \log _{2} n$ for some $k \geq 2$. We obtain that

$$
0 \leq \chi(M) \leq 2^{k}\left(\frac{n}{2}+1\right)
$$

Moreover, if $\chi(M)>0$, then the number of generators $l$ of the rational cohomology algebra $H^{*}(M ; \mathbb{Q})$ is restricted by $1 \leq l \leq k$.

Compare the condition $\chi(M)>0$ and the symmetry assumption to Theorem B.

In [3], we prove that the Euler characteristic is bounded above by a constant multiple of $n \log n$ without the assumption of rational ellipticity. Here we obtain a linear bound, which matches the growth rate of $\chi\left(\mathbb{C P}^{n / 2}\right)=n / 2+1$.

General conventions All cohomology algebras are taken with rational coefficients (unless stated otherwise). All cochain algebras are over the rationals. All manifolds are closed.

Structure of the article In Section 1, we provide necessary background and illustrate the problems under consideration. In Section 2, we prove Theorem D and Corollary C. In Section 3, we give the proof of Theorem B. Section 4 is devoted to formulating several problems on the structure of positively curved manifolds and to reconciling them with the conjecture of Wilhelm. We then prove Theorem A.

Acknowledgements The authors wish to thank Xiaoyang Chen, Karsten Grove, Martin Kerin, and Fred Wilhelm for discussions about the Bott-Grove-Halperin and Wilhelm conjectures. The authors also want to express their gratitude to the referee for helping to improve and correct the exposition in several places. The first author was supported by a research grant of the German Research Foundation. The second author was partially supported by NSF grants DMS-1045292 and DMS-1404670. 


\section{Preliminaries}

As mentioned in the introduction, a prominent conjecture in the field of non-negative curvature is the following.

Conjecture (Bott, Grove and Halperin) A non-negatively curved manifold is rationally elliptic.

Recall that a simply connected topological space $X$ is called rationally elliptic if $\operatorname{dim} \pi_{*}(X) \otimes \mathbb{Q}$ is finite and $\operatorname{dim} H^{*}(X ; \mathbb{Q})$ is finite. In other words, the first condition means that from some degree on all the rational homotopy groups of $X$ vanish. The second condition is trivial on manifolds.

Rationally elliptic spaces satisfy several relations on the degrees of their rational homotopy groups, which we shall make use of in the course of this paper. See [12, page 434] for a summary of these.

There is another classical conjecture which combines nicely with the Bott-GroveHalperin conjecture.

Conjecture (Hopf) A positively curved manifold of even dimension has positive Euler characteristic.

Recall that a space $X$ is positively elliptic, or $F_{0}$, if it is rationally elliptic and has positive Euler characteristic. These positively elliptic spaces have very strict properties; in particular, their odd Betti numbers vanish.

The Bott-Grove-Halperin and Hopf conjectures combine nicely to suggest that an even-dimensional manifold of positive curvature is an $F_{0}$-space. For $F_{0}$-spaces there is the Halperin conjecture, which is one of the central conjectures in rational homotopy theory.

Conjecture (Halperin) Let $F \stackrel{j}{\hookrightarrow} E \rightarrow B$ be a fibration of simply connected spaces with $F$ being $F_{0}$. Then the fibration is rationally totally non-cohomologous to zero, ie the induced homomorphism $j^{*}: H^{*}(E ; \mathbb{Q}) \rightarrow H^{*}(F ; \mathbb{Q})$ is surjective.

Equivalently, the rational Leray-Serre spectral sequence degenerates at the $E_{2}$-term, or the cohomology module of the total space splits as the product of fiber and base cohomologies. 
Several attempts have been made to confirm this conjecture and various special cases have been established (see [12]). For example, the conjecture holds true if the cohomology algebra $H^{*}(F ; \mathbb{Q})$ has at most 3 generators (see Theorem 1.2), if all the generators are of the same degree (see Samuel and Zariski [48; 49]), in the "generic case" (see Papadima and Paunescu [33]), on homogeneous spaces (see Shiga and Tezuka [37]), or if the manifold $M^{2 n}$ admits a hard Lefschetz structure

$$
H^{n-k}(M ; \mathbb{R}) \stackrel{\cong}{\rightrightarrows} H^{n+k}(M ; \mathbb{R}), \quad x \mapsto \omega^{k} \cdot x
$$

for some 2-form $\omega$ such as Kähler manifolds do. This last result was established by Meier [32] (see Blanchard [6] for the original statement). It relies on earlier work of Meier [31], in which he gives a very nice reformulation - which we shall draw on - of the Halperin conjecture in terms of self-homotopy equivalences of $F$ or via negative-degree derivations on the rational cohomology algebra. The result is as follows (see [31, Theorem A, page 329]).

Theorem 1.1 (Meier) An $F_{0}$-space satisfies the Halperin conjecture if and only if its rational cohomology algebra does not permit non-trivial derivations of negative degree.

From this characterization it follows easily that once the cohomology algebra is generated by one element, it satisfies the Halperin conjecture. In fact, using this characterization the next theorem was proved by Lupton [27].

Theorem 1.2 The Halperin conjecture holds for an $F_{0}$-space $M$ if the rational cohomology algebra of $M$ has at most 3 generators.

The importance of Halperin's conjecture surpasses theory-internal interest. For example, the same problem appears in the deformation of singularities (see Wahl [40]) and in the existence question of non-negatively curved metrics (see Belegradek and Kapovitch [4]). For an outline of the Wilhelm conjecture, see Section 4.

\section{Proof of Theorem D and of Corollary C}

In this section we prove Theorem $\mathrm{D}$ and derive Corollary $\mathrm{C}$ on the Halperin conjecture as a consequence. In fact, we prove the following, slightly stronger version of Theorem D.

Theorem 2.1 Let $M^{n}$ be rationally elliptic of positive curvature and with symmetry rank $r \geq n /(2 k)+2 \log _{2} n$ for some positive integer $k$. Then:

(1) The Euler characteristic satisfies $0 \leq \chi(M)<2^{k} \chi\left(\mathbb{C P}^{n / 2}\right)$.

(2) If $\chi(M)>0$, then the number of generators $l$ of the rational cohomology algebra $H^{*}(M ; \mathbb{Q})$ satisfies $l \leq k$, with equality only if the minimal model of $M$ contains the minimal model of $\mathbb{S}^{2}$ as a sub-differential graded algebra. 
Proof First, if $k \leq 3$, it follows from results of Grove and Searle [19] and Wilking [44] (see also [3, Lemma 4.2, page 16]) that $M$ is rationally equivalent to a compact rank one symmetric space. In particular, $0 \leq \chi(M) \leq \chi\left(\mathbb{C P}^{n / 2}\right)$, and the rational cohomology has exactly one algebra generator.

Assume therefore that $k \geq 4$. We first prove the second assertion of the theorem. That is, we assume $\chi(M)>0$, and we show that the number of generators $l$ of the cohomology algebra $H^{*}(M ; \mathbb{Q})$ is bounded above by $k$, with equality only if the minimal model $(\Lambda V, \mathrm{~d})$ for $M$ fits into a rational fibration

$$
\left(\Lambda\left\langle e, e^{\prime}\right\rangle, \mathrm{d} e^{\prime}=e^{2}\right) \hookrightarrow(\Lambda V, \mathrm{~d}) \rightarrow(\Lambda W, \overline{\mathrm{d}})
$$

over an $F_{0}$-algebra $(\Lambda W, \overline{\mathrm{d}})$ (due to [12, Theorem 28.6, page 375]).

Since $M$ is an $F_{0}$ space, there exists a pure model $\left(\Lambda\left\langle x_{1}, \ldots, x_{l}, y_{1}^{\prime}, \ldots, y_{l}^{\prime}\right\rangle\right.$, d) where $\mathrm{d} x_{i}=0, \mathrm{~d} y_{i}^{\prime} \in \Lambda\left\langle x_{1}, \ldots, x_{l}\right\rangle$, the $x_{i}$ have even degree, and the $y_{i}^{\prime}$ have odd degree. Set $y_{i}=\mathrm{d} y_{i}^{\prime}$. We may reindex such that $\operatorname{deg} x_{1} \leq \cdots \leq \operatorname{deg} x_{l}$ and $\operatorname{deg} y_{i} \geq 2 \operatorname{deg} x_{i}$ for all $i$. For this we point the reader to Remark 2.2.

Set $c=\lfloor n / k\rfloor+2$. Observe that the symmetry rank is at least $2 \log _{2} n+c / 2-1$. By [25] we conclude that $H^{*}(M)$ is 4-periodic up to degree $c$. This implies that the truncated rational cohomology algebra $H^{*<c}(\cdot ; \mathbb{Q})$ of $M$ is isomorphic to that of $\mathbb{S}^{\infty}, \mathbb{C} \mathrm{P}^{\infty}, \mathbb{H} \mathrm{P}^{\infty}$, or $\mathbb{S}^{2} \times \mathbb{H} \mathrm{P}^{\infty}$. In the notation above, we have one of the following four cases:

- (Sphere case) $\operatorname{deg} y_{i}-\operatorname{deg} x_{i} \geq \operatorname{deg} x_{i} \geq c$ for all $1 \leq i \leq l$.

- ( $\mathbb{C P}$ case) $\operatorname{deg} x_{1}=2, \operatorname{deg} y_{1} \geq c$, and $\operatorname{deg} y_{i}-\operatorname{deg} x_{i} \geq \operatorname{deg} x_{i} \geq c$ for all $2 \leq i \leq l$.

- $\left(\mathbb{H P}\right.$ case) $\operatorname{deg} x_{1}=4, \operatorname{deg} y_{1} \geq c$, and $\operatorname{deg} y_{i}-\operatorname{deg} x_{i} \geq \operatorname{deg} x_{i} \geq c$ for all $2 \leq i \leq l$.

- $\left(\mathbb{S}^{2} \times \mathbb{H P}\right.$ case $) \operatorname{deg} x_{1}=2, \operatorname{deg} y_{1}=4, \operatorname{deg} x_{2}=4, \operatorname{deg} y_{2} \geq c$, and $\operatorname{deg} y_{i}-$ $\operatorname{deg} x_{i} \geq \operatorname{deg} x_{i} \geq c$ for all $3 \leq i \leq l$.

In the first case, the dimension formula implies

$$
l c \leq \sum\left(\operatorname{deg} y_{i}-\operatorname{deg} x_{i}\right)=n .
$$

Arguing similarly, we conclude in each of the first three cases that $l c \leq n+4$. Since $k \geq 4$ and $c>n / k+1 \geq(n+4) / k$, we have $l \leq(n+4) / c<k$ in each of the first three cases. In the fourth case, the dimension formula implies

$$
(l-1) c-2 \leq \sum\left(\operatorname{deg} y_{i}-\operatorname{deg} x_{i}\right)=n,
$$


hence $l-1 \leq(n+2) / c$. Since $c>(n+2) / k$, this implies $l \leq k$.

As a quick check, we note that these estimates on $l$ can be realized if we do not require $M$ to satisfy the curvature condition. For example, an $n$-dimensional product of $k-1$ spheres of dimensions at least $c=\lfloor n / k\rfloor+2$ realizes the bound $l=k-1$ in the first case. Replacing the first spherical factor by a $\mathbb{C P}^{m}, \mathbb{H} \mathrm{P}^{m}$, or $\mathbb{S}^{2} \times \mathbb{H} \mathrm{P}^{m}$ of the appropriate dimension shows that the estimate is sharp in these cases as well.

Since $l=k$ only occurs in the $\mathbb{S}^{2} \times \mathbb{H P}$ case, and since $\left(\Lambda\left\langle x_{1}, y_{1}^{\prime}\right\rangle, \mathrm{d}\right)$ is a sub-dga of the minimal model of $M$ in this case, this concludes the proof of the first assertion of the theorem.

We proceed to the proof of the first claim, ie $0 \leq \chi(M)<2^{k} \chi\left(\mathbb{C P}^{n / 2}\right)$. The Euler characteristic is zero for odd-dimensional spaces, and $\chi(M) \geq 0$ in general for rationally elliptic spaces, so we assume that $n$ is even and that $\chi(M)>0$. In other words, we assume that $M$ is an $F_{0}$-space and hence admits a pure model as above (see [12, Proposition 32.10, page 144]). We adopt the notation above.

The Euler characteristic $\chi(M)=\chi(H(\Lambda V, \mathrm{~d}))$ does not depend on the differentials in the minimal model, but only on the degrees of the generators $x_{i}$ and $y_{i}^{\prime}$. This follows from the formula

$$
\chi(M)=\prod_{1 \leq i \leq l} \operatorname{deg} y_{i} / \operatorname{deg} x_{i}
$$

from [12, Proposition 32.15 (iii), page 448].

We consider first the sphere case, where $M$ is $(c-1)$-connected. The solution to the following optimization problem gives an upper bound for the Euler characteristic:

$$
\begin{aligned}
\operatorname{maximize} & \prod_{1 \leq i \leq l} \operatorname{deg} y_{i} / \operatorname{deg} x_{i} \\
\text { over } & l, \operatorname{deg} x_{i}, \operatorname{deg} y_{i} \\
\text { subject to } & l \geq 1, \quad \operatorname{deg} y_{i}-\operatorname{deg} x_{i} \geq \operatorname{deg} x_{i} \geq c, \\
& \sum\left(\operatorname{deg} y_{i}-\operatorname{deg} x_{i}\right)=n .
\end{aligned}
$$

We may treat $\chi(M)$ as a function of $\operatorname{deg} x_{i}$ and $\operatorname{deg} y_{i}-\operatorname{deg} x_{i}$. The expression for $\chi(M)$ is decreasing in $\operatorname{deg} x_{i}$, hence the maximum is achieved along the subset with $\operatorname{deg} x_{i}=c$ for all $i$. It follows that

$$
\chi(M) \leq \prod_{i=1}^{l}\left(1+\frac{\operatorname{deg} y_{i}-\operatorname{deg} x_{i}}{c}\right),
$$


where $1 \leq l<k$ and where the $\operatorname{deg} y_{i}-\operatorname{deg} x_{i}$ are at least $c$ and sum to $n$. For a fixed value of $l$, the right-hand side is bounded above by the case where all $\operatorname{deg} y_{i}-\operatorname{deg} x_{i}$ are equal to each other, and hence equal to $n / l$. Hence

$$
\chi(M) \leq\left(1+\frac{n / l}{c}\right)^{l} .
$$

The right-hand side is increasing in $l$. Using the estimates $l \leq k$ and $c>\frac{n}{k}$ we conclude that

$$
\chi(M) \leq\left(1+\frac{n / k}{c}\right)^{k}<2^{k} .
$$

This concludes the proof in this case.

The proofs in the other cases are similar. The extremal case is the $\mathbb{S}^{2} \times \mathrm{HP}$ case, so we sketch it here. After setting up the corresponding optimization problem, we arrive at the bound

$$
\begin{aligned}
\chi(M) & =\left(1+\frac{2}{2}\right)\left(1+\frac{\operatorname{deg} y_{2}-4}{4}\right) \prod_{i=3}^{l}\left(1+\frac{\operatorname{deg} y_{i}-\operatorname{deg} x_{i}}{\operatorname{deg} x_{i}}\right) \\
& \leq 2\left(1+\frac{n-2}{4}\right)\left(1+\frac{n /(l-2)}{c}\right)^{l-2} \leq\left(\frac{n}{2}+1\right)\left(1+\frac{n / k}{c}\right)^{k}<\chi\left(\mathbb{C P}^{n / 2}\right) 2^{k} .
\end{aligned}
$$

Note that we used the dimension formula to conclude that deg $y_{2}-4 \leq n-2$ since $\operatorname{deg} y_{1}-\operatorname{deg} x_{1}=2$.

Remark 2.2 For the convenience of the reader we shall now give a simple argument for the result from the proof of [12, Theorem 32.6, page 443] that we may order the $x_{i}$ and the $y_{i}$ in such a way that

$$
2 \operatorname{deg} x_{i} \leq \operatorname{deg} y_{i} .
$$

Indeed, we may argue as follows: The $y_{i}$ form a regular sequence. Denote by $I(\cdot)$ the ideal generated by specified elements in $\mathbb{Q}\left[x_{1}, \ldots, x_{l}\right]$.

We may assume that the $x_{i}$ are ordered by degree, beginning with the smallest. Now we choose a permutation of the $y_{i}$ according to the following rule: choose $y_{i_{l}}$ such that $y_{i_{l}}$ does not lie in the ideal $I\left(x_{1}, \ldots, x_{l-1}\right)$. Now choose $y_{i_{l-1}}$ - out of the remaining $y_{i}$ - such that $y_{i_{l-1}}$ does not lie in $I\left(x_{1}, \ldots, x_{l-2}\right)$, ie we always have $y_{i_{j}} \notin I\left(x_{1}, \ldots, x_{j-1}\right)$.

Such a choice can always be made, since the $y_{i}$ (and every permutation of them) form a regular sequence. Indeed, assume the contrary. That is, suppose that at some point there is no valid choice of $y_{j}$. This means that all the remaining $(l-j)$ many $y_{i}$ lie completely in the ideal $I\left(x_{1}, \ldots, x_{l-j-1}\right)$. This implies that there is an $\tilde{l}$ with 
$l-j \leq \tilde{l} \leq l$ such that no power of $\left[x_{\tilde{l}}\right]$ vanishes in cohomology; a contradiction to the finite-dimensionality of $H^{*}(X)$.

Consequently, each $y_{i_{j}}$ has a nontrivial summand which is formed of factors out of the $x_{j}, \ldots, x_{l}$ only; the word length in these factors is at least 2 . Since the $x_{i}$ were ordered by degree, we have that $\operatorname{deg} x_{j} \leq \operatorname{deg} x_{j+1} \leq \cdots \leq \operatorname{deg} x_{l}$. This implies that the summand, and thus the entire term $y_{i_{j}}$ satisfies $2 \operatorname{deg} x_{j} \leq \operatorname{deg} y_{i_{j}}$. This proves that the number $l$ of algebra generators of $H^{*}(M)$ satisfies

$$
\chi(M) \geq 2^{l}
$$

provided $\chi(M)>0$. This directly follows from Equation (1) computing the Euler characteristic of an $F_{0}$-space via the degrees of its homotopy groups.

Assuming Theorem 2.1, we reduce Corollary $\mathrm{C}$ to previously known results.

Proof of Corollary C Recall that $M^{n}$ has symmetry rank at least $n / 8+2 \log _{2} n$. Taking $k=4$ in Theorem $\mathrm{D}$, we conclude either that the cohomology of $M$ has at most three generators or that it has four generators and fits into a rational fibration

$$
\left(\Lambda\left\langle e, e^{\prime}\right\rangle, \mathrm{d} e^{\prime}=e^{2}\right) \hookrightarrow(\Lambda V, \mathrm{~d}) \rightarrow(\Lambda W, \overline{\mathrm{d}}) .
$$

In the first case, $M$ satisfies the Halperin conjecture by Theorem 1.2 in the case of at most three generators.

In the second case, the $H(\Lambda W, \overline{\mathrm{d}})$ is also $F_{0}$ (due to [12, Theorem 28.6, page 375]) and has at most three generators. By Lupton's theorem again, it satisfies the Halperin conjecture. Due to [30, Theorem 1, page 154], we conclude that $M$ satisfies the Halperin conjecture.

Remark 2.3 We remark that for a fixed $k$ (not depending on $n$ ) the upper bound lies in $\mathcal{O}(n)$ and is optimal in this sense. For example, taking $k=4$, it follows from Theorem D that symmetry rank at least $n / 8+2 \log _{2} n$ implies

$$
0 \leq \chi(M) \leq 8 n+16
$$

We proceed to a further corollary, which we apply in the proof of Proposition 4.20. 
Corollary 2.4 If $M^{n}$, for $n$ even, is hard Lefschetz, positively elliptic, positively curved and has symmetry rank at least $n / 8+2 \log _{2} n$, then $M$ is either a rational $\mathrm{CP}^{n / 2}$ or has the rational homotopy type given by a minimal model of the form

$$
\begin{aligned}
(\Lambda\langle u, a, x, y\rangle, \mathrm{d}), \quad & \operatorname{deg} u=2, \quad \operatorname{deg} a \geq n / 4+1, \\
& \operatorname{deg} y=n+1-\operatorname{deg} a \neq 2 \operatorname{deg} a-1=\operatorname{deg} x, \\
& \mathrm{~d} u=\mathrm{d} a=0, \\
& \mathrm{~d} x=a^{2}+k_{1} a u^{\operatorname{deg} a / 2}+k_{2} u^{\operatorname{deg} a}, \\
& \mathrm{~d} y=a u^{(n-2 \operatorname{deg} a+2) / 2}+k_{3} u^{(n-\operatorname{deg} a+2) / 2} \quad \text { for } k_{i} \in \mathbb{Q} .
\end{aligned}
$$

Proof Due to Theorem D we know that there are at most 4 generators of the rational cohomology algebra of $M$. The proof of the theorem tells us that, since $M$ looks like $\mathbb{C P}^{\infty}$ in low degrees, the number of generators is at most 3 .

We have to show that there cannot be exactly three algebra generators of $H^{*}(M)$. Suppose $(\Lambda\langle u, a, b, x, y, z\rangle, \mathrm{d})$ with $\operatorname{deg} u=2, \operatorname{deg} a \geq n / 4+1, \operatorname{deg} b \geq n / 4+1$ is the minimal model of $M$. Since $M$ is hard Lefschetz, the dual of an element lies in the ideal generated by $[u]$; in particular, so do the elements $\left[a^{2}\right],\left[b^{2}\right],[a b]$. Thus, due to hard Lefschetz, we conclude that $\operatorname{deg} x=\operatorname{deg} a^{2}-1=2 \operatorname{deg} a-1$, $\operatorname{deg} y=\operatorname{deg} b^{2}-1=2 \operatorname{deg} b-1, \operatorname{deg} z=\operatorname{deg} a b-1=\operatorname{deg} a+\operatorname{deg} b-1$. Using this information we compute the dimension of $M$ in terms of the degrees and we obtain the contradiction

$$
\begin{aligned}
n=\operatorname{dim} M & =(2 \operatorname{deg} a+2 \operatorname{deg} b+\operatorname{deg} a+\operatorname{deg} b)-(2+\operatorname{deg} a+\operatorname{deg} b) \\
& =2 \operatorname{deg} a+2 \operatorname{deg} b-2 \geq 2 \cdot(n / 2+2)-2>n .
\end{aligned}
$$

Thus there may be at most one algebra generator of $H^{*}(M)$ above degree $n / 4$ and the minimal model of $M$ has the form

$$
(\Lambda\langle u, a, x, y\rangle, \mathrm{d}), \quad \operatorname{deg} u=2, \quad \operatorname{deg} a \geq n / 4+1, \quad \mathrm{~d} u=\mathrm{d} a=0 .
$$

Due to hard Lefschetz we derive $\operatorname{deg} x=2 \operatorname{deg} a-1$ and

$$
\mathrm{d} x=a^{2}+k_{1} a u^{\operatorname{deg} a / 2}+k_{2} u^{\operatorname{deg} a} .
$$

Consequently, we have $\operatorname{deg} y=n+1-\operatorname{deg} a$. Thus we have to consider two cases. In the first case $\operatorname{deg} x=\operatorname{deg} y=(2 n+1) / 3, \operatorname{deg} a=(n+2) / 3$ imply that $\left\langle\left[a^{2}\right],\left[a u^{(n+2) / 6}\right],\left[u^{(n+2) / 3}\right]\right\rangle \subseteq H^{(2 n+4) / 3}(M)$ is just 1-dimensional; a contradiction to Poincaré duality. In the second case we derive

$$
\mathrm{d} y=a u^{(n-2 \operatorname{deg} a+2) / 2}+k_{3} u^{(n-\operatorname{deg} a+2) / 2}
$$

due to hard Lefschetz again. 
The given homotopy type includes the one of $\mathrm{SO}(n+2) / \mathrm{SO}(n) \times \mathrm{SO}(2)$. We leave it to the reader to adapt this result to quaternionically hard Lefschetz manifolds. Depending on the degrees of the elements in the minimal model, further restrictions on the $k_{i}$ apply. Clearly, in the case of a cohomologically symplectic $M$ (not necessarily hard Lefschetz) the number of generators of the cohomology algebra still can be at most 3 .

Remark 2.5 Let us remark on a very special case of the theorem. Let $M^{2 n}$ be a positively curved manifold with the rational homotopy type of a simply connected compact symmetric space $N$. If $M$ has symmetry rank at least $2 n / 8+2 \log _{2}(2 n)$, then $N$ is a product of a $\mathbb{C P} \mathrm{P}^{k}, \mathbb{H} \mathrm{P}^{k}, \mathbb{H} \mathrm{P}^{k} \times \mathbb{S}^{2}, \mathbb{S}^{k}, \mathrm{SO}(k+2) / \mathrm{SO}(k) \times \mathrm{SO}(2)$, $\mathrm{SO}(k+3) / \mathrm{SO}(k) \times \mathrm{SO}(3)$ with at most two further spherical factors.

This follows directly from [3, Corollary $\mathrm{D}]$ and that $M$ has to be highly periodic.

Note that on a hermitian symmetric space we need less symmetry.

Proposition 2.6 If $M^{2 n}$ is a positively curved manifold with the rational homotopy type of a simply connected hermitian symmetric space $N$ and if $M$ has symmetry rank at least $2 \log _{2}(2 n)+6$, then $N$ is either $\mathbb{C P}^{n}$ or $\mathrm{SO}(n+2) / \mathrm{SO}(2) \times \mathrm{SO}(n)$.

Proof We make use of the classification of irreducible hermitian symmetric spaces. Such a space is one of

$$
\begin{aligned}
& M_{1}:=\mathrm{SU}(p+q) / \mathrm{S}(U(p) \times U(q)), \\
& M_{2}:=\mathrm{SO}(2 n) / U(n), \\
& M_{3}:=\mathrm{Sp}(n) / U(n), \\
& M_{4}:=\mathrm{SO}(n+2) / \mathrm{SO}(n) \times \mathrm{SO}(2), \\
& M_{5}:=\mathrm{E}_{6} / \mathrm{SO}(10) \times \mathrm{SO}(2), \\
& M_{6}:=\mathrm{E}_{7} / \mathrm{E}_{6} \times \mathrm{SO}(2)
\end{aligned}
$$

For $M_{1}$ we may assume that $p, q \geq 2$, since $M_{1}$ is complex projective otherwise. For $M_{2}$ we may assume that $n>3$ by the classification results in small dimensions. Finally, note that $M_{4}$ appears in the conclusion, so we do not analyze it further.

To exclude the remaining cases, we compute the respective first few Betti numbers as

$$
\begin{aligned}
& M_{1}: \quad b_{2}=1, \quad b_{4}=2, \\
& M_{2}: \quad b_{2}=1, \quad b_{4}=1, \quad b_{6}=2 \text {, } \\
& M_{3}: \quad b_{2}=1, \quad b_{4}=1, \quad b_{6}=2 \text {, } \\
& M_{5}: \quad b_{2}=1, \quad b_{4}=1, \quad b_{6}=1, \quad b_{8}=2 \text {, } \\
& M_{6}: \quad b_{2}=1, \quad b_{4}=1, \quad b_{6}=1, \quad b_{8}=1, \quad b_{10}=2 \text {. }
\end{aligned}
$$


In the last two cases this can be deduced using the degrees of the non-trivial rational homotopy groups - each one-dimensional - of the exceptional spaces $\mathrm{E}_{6}$ and $\mathrm{E}_{7}$ as being $3,9,11,15,17,23$ and 3,11,15, 19,23, 27, 35 respectively (see [23, page 956]).

The symmetry assumptions guarantee 4-periodicity up to degree 14; in particular, in our case, the Betti numbers have to satisfy $b_{2}=b_{4}=b_{6}=b_{8}=b_{10}=1$. Thus we deduce that the only hermitian symmetric spaces which resemble $\mathbb{C P}^{\infty}$ in degrees 2 to 10 are the ones from the assertion. Note that no Euclidean products may arise, since they have $b_{2}>1$. (Clearly, for small $n$ only $\mathbb{C P}^{n}$ may arise.)

Let us end this section by making a speculation. Let $(\Lambda V$, d) be a simply connected elliptic minimal Sullivan algebra. Denote by $V^{\prime}$ its spherical cohomology, ie the subspace of $V$ with vanishing differential. Then does $H(\Lambda V, \mathrm{~d}) \geq 2^{\operatorname{dim} V^{\prime}}$ hold (with equality if and only if the algebra is isomorphic to the product of $\operatorname{dim}\left(V^{\prime}\right)$ spheres)?

This inequality in the case of (simply connected) $F_{0}$-spaces is classical and the content of Equation (3). Clearly, in this case the cohomology algebra $H^{*}(X)$ has exactly $\frac{1}{2} \operatorname{dim} V$ many algebra generators which correspond exactly to the space $V^{\prime}$.

\section{Proof of Theorem B}

In this section we shall prove the Hopf conjecture for rationally elliptic Riemannian manifolds with logarithmic symmetry rank.

Lemma 3.1 Let $M^{n}$, with $n \geq 8$ even, be a simply connected rationally elliptic space with four-periodic rational cohomology. Then $\chi(M)>0$ and $H^{\text {odd }}(M)=0$.

Proof By definition (see [24]), it suffices to show that $b_{3}(M)=0$. Moreover, when $n \equiv 2 \bmod 4$, the definition together with Poincaré duality and the graded commutativity of the cross product implies that $b_{3}(M)$ is even.

First, assume that $n \equiv 0 \bmod 4$. Due to four-periodicity and Poincaré duality we conclude that $b_{3}(M)=b_{n-1}(M)=0$. Assume therefore that $n \equiv 2 \bmod 4$. Moreover, assume that $M$ is not a rational sphere. It follows from four-periodicity and Poincaré duality that $b_{2}(M)=b_{4}(M)=1$ and

$$
\chi(M)=2+\frac{n-2}{4}\left(2-b_{3}(M)\right) .
$$

Since $M$ is rationally elliptic, we know that $\chi(M) \geq 0$. Combining this with Equation (5) and the estimate $n \geq 10$, we have that $b_{3}(M) \leq 3$. However $b_{3}(M)$ is even, so $b_{3}(M) \leq 2$. Equation (5) now implies $\chi(M)>0$, so by rational ellipticity again, $H^{\text {odd }}(M)=0$. 
Remark 3.2 For the sake of completeness we discuss the Euler characteristic of a six-dimensional simply connected rationally elliptic space.

Since $M$ is simply connected, we derive in this case that $\operatorname{dim} \pi_{3}(M) \otimes \mathbb{Q} \geq b_{3}(M)$. By [12, Theorem 32.14 (iii)], we conclude that

$$
11=2 \operatorname{dim}(M)-1 \geq 3 \operatorname{dim}\left(\pi_{3}(M) \otimes \mathbb{Q}\right) \geq 3 b_{3}(M) .
$$

Moreover, due to Poincare duality, the intersection form is non-degenerate and skewsymmetric, so $b_{3}(M)$ is even. Together, these facts imply that $b_{3}(M) \leq 2$.

Clearly, $\chi(M)>0$ if $b_{3}(M)=0$, so assume that $b_{3}(M)=2$. In this case, the dimension formula (see [12, Theorem 32.14 (i)]) implies that $\pi_{2}(M) \otimes \mathbb{Q}=0$. Altogether, either $\chi(M)>0$ or $M$ has the rational type of $\mathbb{S}^{3} \times \mathbb{S}^{3}$.

Proof of Theorem B It suffices to prove that $\chi(M)>0$. Indeed, a rationally elliptic space $M$ of positive Euler characteristic has vanishing odd-degree Betti numbers; see [12, Proposition 32.10, page 444]. Poincaré duality then implies $\chi(M) \geq 2$.

We mimic the proof of [3, Theorem A]. Recall our notation from there. Let $T$ be a torus acting effectively on $M$. If $N \subseteq M$ is a submanifold on which $T$ acts, we set $\operatorname{dk}(N):=\operatorname{dim} \operatorname{ker}\left(\left.T\right|_{N}\right)$. We also denote by $\operatorname{cod}(N)$ the codimension of $N$. Recall that $M_{x}^{\iota}$ denotes the fixed-point component of the involution $\iota$ at the fixed-point $x \in M$.

We proceed by induction over the dimension. If $n \leq 48$, the symmetry assumption is larger than $n / 4+1$ (and larger than $n / 2$ for $n<10$ ), hence the result follows from the homotopy classification (resp. the diffeomorphism classification) of Wilking [44] (resp. Grove and Searle [19]). Therefore let $n \geq 50$.

As in [3], we have to differentiate two cases. Consider the isotropy representation of the isometrically acting torus at a fixed-point $x$.

Case 1 There exists $x \in M^{T}$ such that every $\iota \in \mathbb{Z}_{2}^{\operatorname{dim} T}$ with $\operatorname{dk}\left(M_{x}^{\iota}\right) \leq 1$ and $\operatorname{cod}\left(M_{x}^{\iota}\right) \leq n / 2$ actually has $\operatorname{cod}\left(M_{x}^{\iota}\right) \leq n / 4$.

Case 2 For all $x \in M^{T}$, there exists $\iota \in \mathbb{Z}_{2}^{\operatorname{dim} T}$ with $\operatorname{dk}\left(M_{x}^{\iota}\right) \leq 1$ and $n / 4<$ $\operatorname{cod}\left(M_{x}^{\iota}\right) \leq n / 2$.

In the first case, we conclude in [3, Proof of Theorem A] that $M$ has 4-periodic cohomology, and Lemma 3.1 yields the result. Hence we may assume that Case 2 applies.

Cover the torus fixed-point set by the respective $M_{x}^{\iota}$ from Case 2. We use the fact that the fixed-point set of a circle action on a rationally elliptic compact manifold is again rationally elliptic (see [1, Corollary 3.3.11, page 155]). We observe the following: 
- If $\mathrm{dk}\left(M_{x}^{\iota}\right)=0$, we consider the induced, effective action of the torus $\bar{T}=$ $T / \operatorname{ker}\left(\left.T\right|_{M_{x}^{\iota}}\right)$ on $M_{x}^{\iota}$. Note that $\operatorname{dim}(\bar{T})=\operatorname{dim}(T)$. Choose a circle $\mathbb{S}^{1} \subseteq \bar{T}$ together with a fixed-point component $F \subseteq\left(M_{x}^{\iota}\right)^{\mathbb{S}^{1}}$ satisfying $x \in F$ and $\operatorname{dk}(F)=1$. It follows that $F$ is rationally elliptic and has symmetry rank at least $\log _{4 / 3}(\operatorname{dim} F)$. The inductive assumption applies to $F$, so $H^{\text {odd }}(F)=0$.

- If $\operatorname{dk}\left(M_{x}^{\iota}\right)=1$ and $M_{x}^{\iota}$ is a fixed-point component of a circle in $T$, then $M_{x}^{\iota}$ is rationally elliptic. The inductive hypothesis applies to $M_{x}^{\iota}$, so $H^{\text {odd }}\left(M_{x}^{\iota}\right)=0$.

- If $\operatorname{dk}\left(M_{x}^{\iota}\right)=1, M_{x}^{\iota}$ is fixed by a circle $\mathbb{S}^{1}$, but $M_{x}^{\iota}$ is strictly contained in $M_{x}^{\mathbb{S}^{1}}$, then replace $\iota$ by the involution in $\mathbb{S}^{1}$ and proceed as in one of the previous cases.

In this manner we cover the whole fixed-point set $M^{T}$ with involutive fixed-point components $M_{x}^{\iota}$ with vanishing odd Betti numbers. Since the sum of the odd Betti numbers of a component of $M^{T}$ is smaller than or equal to the sum of the odd Betti numbers of the respective $M_{x}^{\iota}$ around it, we have $b_{\text {odd }}\left(M^{T}\right)=0$. Since $\chi(M)=$ $\chi\left(M^{T}\right)>0$, we conclude from rational ellipticity that $H^{\text {odd }}(M)=0$.

Remark 3.3 Suppose $M^{n}$ is a closed, one-connected, rationally elliptic manifold. If $M$ admits an effective action by a $T=\mathbb{S}^{1}$ such that $M^{T}$ has an isolated fixed point, then $\chi(M)>0$, ie $M$ has vanishing odd Betti numbers and $\chi(M) \geq 2$. Indeed, let $F_{0}, F_{1}, \ldots, F_{r}$ be the connected components of $M^{T}$ with $F_{0}$ being an isolated point. Every $F_{i}$ is rationally elliptic and satisfies $\chi\left(F_{i}\right) \geq 0$. We deduce that $\chi(M)=$ $\chi\left(M^{T}\right)=1+\chi\left(F_{1}\right)+\cdots+\chi\left(F_{r}\right)>0$.

\section{On a conjecture of Wilhelm, and the proof of Theorem A}

It is desirable to try to determine the rational homotopy type of a rationally elliptic manifold with positive curvature and symmetry. However, at this stage every approach even using equivariant rational homotopy theory — seems to be doomed to fail unless the following question - admittedly formulated in a suggestive way and vastly generalizing the Hopf conjecture for $\mathbb{S}^{2} \times \mathbb{S}^{2}$ - is answered at least in part.

Question Does positive curvature obstruct the existence of rational product structures?

One positive answer to this question under suitable conditions would show that the rational cohomology algebra cannot split into products. Clearly, this question cannot be answered in the affirmative without further restrictions, since already the Aloff-Wallach spaces split rationally as $\mathbb{S}^{2} \times \mathbb{S}^{5}$. 
Note that a reason that we could make progress on the Halperin conjecture in the case of positive curvature lies in the following fact: the Halperin conjecture is clearly a conjecture on the structure of the cohomology algebra of a space, yet it seems not to differ between products and "non-products", since it holds in the somehow extremal cases of Cartesian products as well as hard Lefschetz manifolds.

In this section, we first discuss the Wilhelm conjecture and several further problems and refinements related to the main question above. This can be seen as a prelude to the second part of this section. The latter is devoted to the proof of Theorem A, in which we establish a generalized version of the Wilhelm conjecture in some cases.

(For the reader familiar with the Wilhelm conjecture, the first part is not necessary for understanding the proofs.)

\subsection{Splitting problems and the Wilhelm conjecture}

Let us discuss different, more precise versions of the main question above and relate them to the following conjecture commonly attributed to Fred Wilhelm.

Conjecture 4.1 (Wilhelm) Let $M \rightarrow B$ be a (non-trivial) Riemannian submersion, with $M$ a complete positively curved manifold. Then $2 \operatorname{dim} B>\operatorname{dim} M$.

In the following we want to assume all positively curved manifolds to be compact. If $M$ is not compact, Walschap proved that there are no non-trivial submersions from $M$ (see [42, Theorem 2.1]). Then the submersion is a fiber bundle (with fiber $F$ ) according to Ehresmann and the conjecture can be stated as $\operatorname{dim} B>\operatorname{dim} F$.

We are grateful to Martin Kerin for bringing the examples $M^{13}=\mathbb{S}^{1} \backslash\left(\mathbb{S}^{7} \times \mathbb{S}^{7}\right)$ and $N^{11}=\mathbb{S}^{3} \backslash\left(\mathbb{S}^{7} \times \mathbb{S}^{7}\right.$ ) to our attention (see [26]). These admit positive curvature almost everywhere, and they fit into Riemannian submersions over $\mathbb{C P}^{2}$ and $\mathbb{S}^{4}$, respectively. The fiber in each case is $\mathbb{S}^{7}$. Moreover, the induced metric (ie the submersion metric) on the base is positively curved. These examples show that, as for the Wilhelm conjecture, the assumption of positive curvature on the total space cannot be weakened to positive curvature almost everywhere (even if one retains the assumption that the base is positively curved).

If two fibers are totally geodesic, the conjecture follows directly from Frankel's result. In the case when all fibers are totally geodesic an even better estimate holds due to Florit and Ziller (see [14], cf [9]).

This conjecture seems to be very hard to tackle. Nonetheless, in this section we shall even generalize this question to the following. 
Question 4.2 Let $M$ be a closed simply connected manifold admitting a metric of positive curvature. If $M$ fits into a (non-trivial rational) fibration $F \hookrightarrow M \rightarrow B$ of simply connected manifolds (or topological spaces of finite type and finite formal dimension), when does $\operatorname{dim} B>\operatorname{dim} F$ hold?

Note that this question can only be answered in the positive under suitable conditions, since the Aloff-Wallach spaces rationally split as a product of $\mathbb{S}^{2}$ and $\mathbb{S}^{5}$.

We want to state the following problems and suggest them as yet another generalized form of the Hopf conjecture on $\mathbb{S}^{2} \times \mathbb{S}^{2}$. Assuming parts of them, we shall try to partially answer Question 4.2. We shall justify this below.

Problem 4.3 Let $M^{n}$ be a simply connected closed manifold admitting a metric of positive sectional curvature. Under what conditions do the following claims hold?

- If $n$ is even, then rationally $M$ does not split as a non-trivial product.

- If $n$ is odd, then rationally $M$ is a formal elliptic pure space with the dimension of the odd degree spherical rational homotopy equal to one, ie the minimal model of $M$ splits as the product of a pure elliptic algebra of positive Euler characteristic and a factor which is the minimal model of an odd-dimensional sphere.

Let us put the second part of this problem in a more general context as far as "splitting properties" are considered. More precisely, we do not content ourselves with potential non-existence of product structures. We are also interested in the question of to what degree splittings in form of (rational) fibrations are possible.

Problem 4.4 Under what conditions is it rationally impossible for $M$ to split as a non-trivial fibration? In other words, for what $M$ does the following hold. If

$$
(\Lambda V, \mathrm{~d}) \hookrightarrow(\Lambda(V \oplus W), \mathrm{d}) \rightarrow(\Lambda W, \overline{\mathrm{d}})
$$

is a rational fibration of Sullivan algebras and $(\Lambda(V \oplus W), \mathrm{d})$ is a model for $M$, then: Weak version $(\Lambda(V \oplus W), \mathrm{d})$ is not minimal as a Sullivan algebra.

Strong version The rational transgression $\mathrm{d}_{0}: W^{*} \rightarrow V^{*+1}$ in the long exact homotopy sequence of the fibration is injective on $W^{\text {odd }}$.

Alternate version With $\left(x_{i}\right)$ a basis of $W^{\text {odd }}$ and $\left(x_{i}^{\prime}\right)$ a basis of $V^{\text {odd }}$, the following hold:

- $\sum \operatorname{deg} x_{i}<\sum \operatorname{deg} x_{i}^{\prime}$.

- The projections $\left.\mathrm{d}\left(x_{i}\right)\right|_{\Lambda V}$ form a regular sequence in $\Lambda V^{\text {even }}$ with the property that $\left.\mathrm{d}(\Lambda V) \cap \mathrm{d}\left(W^{\text {odd }}\right)\right|_{V}=0$. 
(For the rational transgression see [12, page 214].)

No transition of positive curvature to topology seems to be known (from a rational point of view) which is as good as the transition of non-negative curvature to rationally elliptic spaces. Problems 4.3 and 4.4 try to fill this gap by introducing reasonable structures which might be satisfied by positively curved manifolds.

Let us now discuss both problems, first in two separate remarks. First, note that the problems clearly do not imply that $M$ cannot be the total space of a fibration in any case. The low-dimensional examples of positive curvature clearly do admit rational fibration structures. We shall give concrete depictions below.

As to Problem 4.3, we make the following remark.

Remark 4.5 A rationally elliptic space of positive Euler characteristic is formal, since its cohomology is a complete intersection algebra, a quotient of a polynomial algebra in even degrees by a regular sequence (see [12, Proposition 32.10, page 444, and Proposition 32.3, page 437]. Thus a combined Bott-Grove-Halperin-Hopf conjecture would yield the formality of even-dimensional, simply connected, positively curved manifolds.

It is tempting to conjecture this for the odd-dimensional examples too. Indeed, the known examples are formal. Moreover, the main source of examples of positively curved manifolds are biquotients and homogeneous spaces, in particular. Biquotients, however, have the remarkable property that they admit so-called pure (minimal) Sullivan models (see [12, page 435] and [13, Section 3.4.2]), ie particularly simple Sullivan models.

An elliptic pure algebra necessarily splits in the form given in Problem 4.3, if it is formal (see [2, Proposition 3.31, page 114]). Motivated by the known examples of positively curved manifolds, in Problem 4.3 we then additionally suppose that the homotopy Euler characteristic of $M$ is $\chi_{\pi}(M)=1$, which finally leads to the speculated form. Indeed, the vanishing of the homotopy Euler characteristic is equivalent to the positivity of the Euler characteristic (see [12, Proposition 32.10, page 444]) - yielding that $\chi_{\pi}$ vanishes on the positively elliptic factor - and $\chi_{\pi}$ is clearly additive in products.

Remark 4.6 We now discuss Problem 4.4 and its hierarchy.

- Problem 4.4 is obviously related to Problem 4.3. However, note that it is not per se making stronger statements. For example, it does not keep track of the speculation that $\chi_{\pi}(M)$ might equal 1 in the odd-dimensional case or that necessarily pure models should occur. 
- The weak version already generalizes the classical Hopf conjecture on $\mathbb{S}^{2} \times \mathbb{S}^{2}$, ie in particular, no product structures may appear in rational homotopy.

- Since there are no other examples known, one is tempted to directly conjecture that positively curved manifolds of sufficiently large dimension are homotopically simple, like rational CROSSes. We introduced the alternate version to show that a less restrictive, reasonable, and more involved conjecture can be stated. In Example 4.7 we shall show that this version from a rational viewpoint is not good enough to deduce the Wilhelm conjecture.

- The strong version is a special case of the alternate one, where the regular sequence is given by the trivial one consisting of a subset of a basis of $W^{\text {even }}$. The weak version does not a priori imply the strong version. For this consider Example 4.8.

Example 4.7 (alternate version does not imply Wilhelm conjecture) The alternate version of Problem 4.4 does not imply the Wilhelm conjecture purely from a rational viewpoint:

Consider the following rational fibration. In the notation from above, set $V:=$ $\left\langle y_{1}, y_{2}, x_{1}^{\prime}, x_{2}^{\prime}\right\rangle$ with $\operatorname{deg} y_{1}=\operatorname{deg} y_{2}=4, \operatorname{deg} x_{1}^{\prime}=\operatorname{deg} x_{2}^{\prime}=39, \mathrm{~d} y_{1}=\mathrm{d} y_{2}=0$, $\mathrm{d} x_{i}^{\prime}=y_{i}^{10}$. Set $W:=\left\langle x_{1}, x_{2}\right\rangle$ with $\operatorname{deg} x_{1}=27, \operatorname{deg} x_{2}=47, \mathrm{~d} x_{1}=y_{1}^{7}+y_{2}^{7}$, $\mathrm{d} x_{2}=y_{1}^{6} y_{2}^{6}$, where $\mathrm{d}$ is already the twisted differential in the rational fibration. We compute that $\operatorname{deg} x_{1}+\operatorname{deg} x_{2}=74<78=\operatorname{deg} x_{1}^{\prime}+\operatorname{deg} x_{2}^{\prime}$ and $\operatorname{dim}|(\Lambda W, \overline{\mathrm{d}})|=74>$ $72=\operatorname{dim}|(\Lambda V, \mathrm{~d})|$.

Evidently, the strong version of the conjecture implies the weak one. (For this it suffices to note that the rational transgression identifies with the linear part, $\mathrm{d}_{0}$, of the twisted differential, $\mathrm{d}$, as our notation already suggests. The injectivity of $\mathrm{d}_{0}$, then, in particular, shows that $\mathrm{d}$ is not decomposable.) The converse, however, is not true.

Example 4.8 (Weak version does not imply strong version) Set $V:=\left\langle c, x^{\prime}\right\rangle$ with $\operatorname{deg} c=4, \operatorname{deg} x^{\prime}=7, \mathrm{~d} c=0, \mathrm{~d} x^{\prime}=c^{2}$. Set $W:=\left\langle y_{1}, y_{2}, x_{1}, x_{2}\right\rangle$ with $\operatorname{deg} y_{1}=$ $\operatorname{deg} y_{2}=2, \operatorname{deg} x_{1}=\operatorname{deg} x_{2}=3, \mathrm{~d} y_{1}=\mathrm{d} y_{2}=0, \mathrm{~d} x_{1}=y_{1}^{2}+y_{2}^{2}, \mathrm{~d} x_{2}=y_{1} y_{2}+c$ where $\mathrm{d}$ is already the twisted differential in the rational fibration. We compute a minimal model of the total space as $\left(\Lambda\left\langle y_{1}, y_{2}, x_{1}, x^{\prime}\right\rangle, \mathrm{d}\right)$ with $\mathrm{d} y_{i}=0, \mathrm{~d} x_{1}=y_{1}^{2}+y_{2}^{2}$, $\mathrm{d} x^{\prime}=y_{1}^{2} y_{2}^{2}$. This minimal model does not split as a fibration in the sense of the weak conjecture. However, in the sense of the strong conjecture, the transgression $d_{0}$ in the fibration vanishes on $x_{2}$.

This shows that the weak version does not imply the strong one a priori. 
In contrast to the previous observations, we observe that the strong version is good enough to deduce the Wilhelm conjecture.

Lemma 4.9 Let $F \hookrightarrow E \rightarrow B$ be a (non-trivial) fibration of simply connected, rationally elliptic spaces. Suppose the strong conclusion of Problem 4.4 holds for $E$ (not necessarily admitting positive curvature). Then it holds that $\operatorname{dim} F<\operatorname{dim} B$.

Proof We compute the dimension of $B$ using the cohomological information of $F$. That is, let $x_{1}, \ldots, x_{n}$ be a basis of $W^{\text {odd }}$ where $(\Lambda W, \overline{\mathrm{d}})$ is the minimal model of $F$. It is trivial to see using the dimension formula (see [12, page 434]) that

$$
\operatorname{dim} F=\operatorname{dim}|(\Lambda W, \overline{\mathrm{d}})| \leq \operatorname{dim} \prod_{1 \leq i \leq n} \mathbb{S}^{\operatorname{deg} x_{i}}=\sum_{1 \leq i \leq n} \operatorname{deg} x_{i} .
$$

According to Problem 4.4, the rational transgression on $W^{\text {odd }}$ is injective, ie there is an $n$-dimensional (not necessarily homogeneous) subspace $\left\langle y_{1}, \ldots, y_{n}\right\rangle \subseteq V^{\text {even }}$ where $(\Lambda V, \mathrm{~d})$ is the minimal model of $B$. Since $(\Lambda V, \mathrm{~d})$ is rationally elliptic, it has finite dimension. Consequently, we obtain that $\operatorname{dim} V^{\text {odd }} \geq n$. As in the proof of Theorem $\mathrm{D}$ we may order a basis $\left(z_{i}\right)_{1 \leq i \leq n^{\prime}}$ of $V^{\text {odd }}$ such that $\operatorname{deg} z_{i} \geq 2 \operatorname{deg} y_{i}$ for $1 \leq i \leq n$. As a consequence, we derive that

$$
\operatorname{dim} B=\operatorname{dim}|(\Lambda V, \mathrm{~d})| \geq \prod_{1 \leq i \leq n} \mathbb{S}^{\operatorname{deg} y_{i}}=\sum_{1 \leq i \leq n} \operatorname{deg} y_{i}=\left(\sum_{1 \leq i \leq n} \operatorname{deg} x_{i}\right)+n
$$

(For this estimate we use [12, Theorem 32.6 (ii), page 441], which gives the sum of all even-degree generators as a lower bound on the dimension.)

Comparing this to the estimate of Equation (6) proves the result.

Remark 4.10 We observe that:

- The estimate $\operatorname{dim} B-\operatorname{dim} F \geq n$ from the lemma is sharp, as the example $\mathbb{S}^{3} \hookrightarrow T_{1} \mathbb{S}^{4} \rightarrow \mathbb{S}^{4}$ shows. From [20] and [10] we recall that there exists a space homeomorphic to the total space in this case which does carry positive curvature.

- If $F$ is $F_{0}$ we may improve the estimate on its dimension by

$$
\operatorname{dim} F \leq \operatorname{dim} \prod_{1 \leq i \leq n} \mathbb{C P}^{\left(\operatorname{deg} x_{i}-1\right) / 2}=\left(\sum_{1 \leq i \leq n} \operatorname{deg} x_{i}\right)-n,
$$

and the gap in $\operatorname{dimension}$ is $\operatorname{dim} B-\operatorname{dim} F \geq 2 n$. Also this estimate is sharp due to $\mathbb{C P}^{1} \hookrightarrow \mathbb{C P}^{2} \rightarrow \mathbb{H} \mathrm{P}^{1}$, the twistor submersion over the positive quaternion Kähler manifold $\mathbb{H} \mathrm{P}^{1}$ of positive curvature (with $\mathbb{C P}^{2}$ carrying positive curvature). 
As we remarked we suggest Problem 4.4 as a rational version of the Wilhelm conjecture. That is, transitioning positive curvature as rationally elliptic to the world of rational spaces, the strong version of Problem 4.4 implies the Wilhelm conjecture.

Theorem 4.11 Assume the Bott-Grove-Halperin conjecture as well as the strong version of Problem 4.4 to be true. It follows that the Wilhelm conjecture holds true.

Proof If the total space $M^{n}$ of a Riemannian submersion is positively curved, so is the base space, due to O'Neill. Due to the Bott-Grove-Halperin conjecture, both spaces are rationally elliptic. The long exact sequence of the fibration tells us that also the fiber is rationally elliptic. Lemma 4.9 yields the result.

\subsection{Proofs}

Let us now head towards the proof of Theorem A. For this we shall need the following recent result (see [47, page 10] and [22]).

Theorem 4.12 (Halperin) Let $F \hookrightarrow X \rightarrow B$ be a fibration where $X$ is a rationally elliptic nilpotent space. Suppose that $B$ is simply connected, rationally of finite type, and that cat $B<\infty$. If the Betti numbers $\operatorname{dim} H_{i}(F)$ grow at most polynomially in $i$, then $B$ is rationally elliptic.

We remark that for our purposes [21, Theorem 4.15(iv), page 207] is fully sufficient.

The proof of Theorem A now splits into various separate theorems (Theorems 4.13, 4.17, and 4.19), which actually prove a stronger version. That is, they confirm Question 4.2 in the respective cases.

Theorem 4.13 Let $M$ be simply connected with singly generated rational cohomology algebra. If $M$ is the total space of a (rational) fibration of simply connected spaces of finite type and finite formal dimension $F \hookrightarrow M \rightarrow B$, then $\operatorname{dim} B>\operatorname{dim} F$.

Proof In this case $M$ is necessarily rationally elliptic. Consider a fibration

$$
F \stackrel{j}{\hookrightarrow} M \stackrel{p}{\rightarrow} B .
$$

We assume the fibration to be non-trivial.

Since the fiber cohomology is finite-dimensional and since a finite $\mathrm{CW}$-complex has finite Lusternik-Schnirelmann category (see [12, Proposition 27.5, page 354]), we may apply Theorem 4.12 and the long exact homotopy sequence of a fibration to deduce that all three of $F, M$ and $B$ are rationally elliptic. 
We now distinguish two cases. Either the rational cohomology of $M$ is concentrated in even degrees, or $M$ is a rational odd-dimensional sphere.

Case 1 In this case the Euler characteristic of $M$ is positive. Since the Euler characteristic is multiplicative, both $\chi(F)>0$ and $\chi(B)>0$. This implies that both $F$ and $B$ are $F_{0}$ spaces (with rational cohomology concentrated in even degrees). Consequently, the associated Leray-Serre spectral sequence degenerates at the $E_{2}$-term and

$$
H^{*}(M) \cong H^{*}(B) \otimes H^{*}(F)
$$

as a module. In particular, $j^{*}: H^{*}(M) \rightarrow H^{*}(F)$ is surjective.

Write $H^{*}(M ; \mathbb{Q}) \cong \mathbb{Q}[u] /\left(u^{n+1}\right)$ for some $n \geq 1$. It follows that $v=j^{*}(u)$ generates $H^{*}(F ; \mathbb{Q})$. Choose $m \geq 1$ such that $H^{*}(F ; \mathbb{Q}) \cong \mathbb{Q}[v] /\left(v^{m+1}\right)$. By Equation $(7)$, the space $B$ is rationally $m \operatorname{deg}(u)$-connected. Since $B \not \mathbb{Q} *$, it follows that $\operatorname{dim}(B)>$ $m \operatorname{deg}(u)=\operatorname{dim}(F)$, as claimed.

Case 2 Suppose $M \cong \mathbb{Q} \mathbb{S}^{k}$ with $k>1$ odd. As in Case 1 , let $(\Lambda V, \mathrm{~d})$ and $(\Lambda W, \overline{\mathrm{d}})$ denote minimal models of $B$ and $F$, respectively. Since $F$ and $B$ are rationally elliptic, there exist bases

- $\left\{a_{i}\right\}_{1}^{l}$ for $V^{\text {even }}$ and $\left\{a_{i}^{\prime}\right\}_{1}^{l^{\prime}}$ for $V^{\text {odd }}$ such that $l \leq l^{\prime}$,

- $\left\{b_{i}\right\}_{1}^{m}$ for $W^{\text {even }}$ and $\left\{b_{i}^{\prime}\right\}_{1}^{m^{\prime}}$ for $W^{\text {odd }}$ such that $m \leq m^{\prime}$.

We identify $V$ and $W$ with the duals of homotopy groups of $B$ and $F$, respectively. Since $M$ is a rational sphere of odd dimension, all of its rational homotopy groups vanish except for $\pi_{k}(M) \otimes \mathbb{Q} \cong \mathbb{Q}$. In particular, the maps $\pi_{i}(F) \otimes \mathbb{Q} \rightarrow \pi_{i}(M) \otimes \mathbb{Q}$ are trivial for all $i \neq k$, and the map $\pi_{k}(F) \otimes \mathbb{Q} \rightarrow \pi_{k}(M) \otimes \mathbb{Q}$ is either trivial or surjective. We consider these two subcases separately.

Case 2.1 Suppose $\pi_{k}(F) \rightarrow \pi_{k}(M)$ is surjective. By dualizing the long exact homotopy sequence and identifying $V^{*} \cong \operatorname{Hom}\left(\pi_{*}(B), \mathbb{Q}\right)$ and $W^{*} \cong \operatorname{Hom}\left(\pi_{*}(F), \mathbb{Q}\right)$, it follows that the rational transgression satisfies

- $\mathrm{d}_{0}: W^{\text {even }} \rightarrow V^{\text {odd }}$ is an isomorphism,

- $\mathrm{d}_{0}: W^{\text {odd }} \rightarrow V^{\text {even }}$ is surjective with one-dimensional kernel.

In terms of the bases chosen above, this implies that

$$
l^{\prime}=m \quad \text { and } \quad m^{\prime}=l+1 .
$$

Moreover, by a homogeneous change of basis, we may assume that

- $\mathrm{d}_{0}\left(b_{i}^{\prime}\right)=a_{i}$ for $1 \leq i \leq l$,

- $\mathrm{d}_{0}\left(b_{i}\right)=a_{i}^{\prime}$ for all $1 \leq i \leq m$,

- $\operatorname{deg}\left(b_{l+1}^{\prime}\right)=k$. 
By the dimension formula, we have

$$
\begin{aligned}
& \operatorname{dim} B=\sum_{1}^{m} \operatorname{deg} a_{i}^{\prime}-\sum_{1}^{l}\left(\operatorname{deg} a_{i}-1\right), \\
& \operatorname{dim} F=\sum_{1}^{l+1} \operatorname{deg} b_{i}^{\prime}-\sum_{1}^{m}\left(\operatorname{deg} b_{i}-1\right) .
\end{aligned}
$$

Adding these together, we get the dimension of the total space:

$$
\begin{aligned}
k & =\operatorname{dim} B+\operatorname{dim} F \\
& =\sum_{1}^{m}\left(\operatorname{deg} a_{i}^{\prime}-\left(\operatorname{deg} b_{i}-1\right)\right)+\sum_{1}^{l}\left(\operatorname{deg} b_{i}^{\prime}-\left(\operatorname{deg} a_{i}-1\right)\right)+\operatorname{deg} b_{l+1}^{\prime} \\
& =\sum_{1}^{m}(2)+\sum_{1}^{l}(0)+k,
\end{aligned}
$$

so $m=0$. Since $l \leq l^{\prime}=m=0$, we have that $B \simeq \mathbb{Q} *$, a contradiction.

Case 2.2 The map $\pi_{k}(F) \rightarrow \pi_{k}(M)$ is trivial. By a similar argument, it follows that

$$
l^{\prime}=m+1 \text { and } m^{\prime}=l .
$$

Similarly, one computes in this case that

$$
k=\operatorname{dim} B+\operatorname{dim} F=\sum_{1}^{l}(0)+\sum_{1}^{m}(2)+k,
$$

so $m=0$. Since $F \Varangle_{\mathbb{Q}} *$, we conclude that

$$
1 \leq m^{\prime}=l \leq l^{\prime}=m+1=1,
$$

and hence that

$$
m^{\prime}=l=l^{\prime}=1
$$

Hence $F$ is a rational sphere of some dimension $2 n-1$, and $B$ is a space with rational cohomology generated by a single element of degree $2 n$. In particular,

$$
\operatorname{dim}(F)=2 n-1<2 n \leq \operatorname{dim}(B) .
$$

An extension of the above argument in Case 2 shows that if $M$ has the rational type of a product of odd-dimensional spheres, then so does $F$. This result is proved in Halperin [21]. With regard to Question 4.2, we obtain an affirmative answer in the special case where $M \simeq \mathbb{Q} \mathbb{S}^{k} \times \mathbb{S}^{k}$. 
Corollary 4.14 Let $M$ be a simply connected space of finite type with $H^{*}(M ; \mathbb{Q})=$ $\Lambda\langle x, y\rangle$, where $\operatorname{deg} x=\operatorname{deg} y$ is odd. Any fibration of simply connected spaces $F \hookrightarrow$ $M \rightarrow B$ satisfies $\operatorname{dim} F \leq \operatorname{dim} B$, with equality only if $F \simeq_{\mathbb{Q}} B \simeq_{\mathbb{Q}} \mathbb{S}^{k}$ and $M$ is rationally a product of these.

Proof The proof proceeds in exactly the same way as the proof of the theorem, using the analogous arguments.

Since the fibration is nontrivial, $x$ and $y$ cannot both come from the fiber. Whenever both $x$ and $y$ come from the base we compute that $\operatorname{dim} B>\operatorname{dim} F$. Assume then that exactly one of $x$ or $y$ comes from the fiber. If $F \simeq \mathbb{Q} \mathbb{S}^{k}$, then so is $B$. Otherwise,

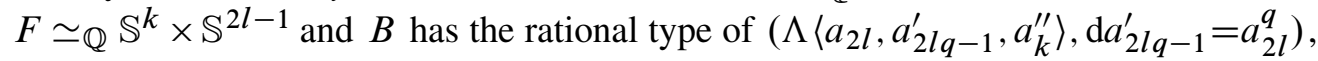
where again subscripts denote degrees, and the remaining differentials vanish. Clearly, $\operatorname{dim} F<\operatorname{dim} B$ in this case.

Note that this result is optimal from a rational point of view within the class of spaces $M$ with freely generated rational cohomology. Indeed, $M$ can arise from products of singly generated spaces of arbitrary odd dimension.

In the next corollary we apply these algebraic results to confirm the Wilhelm conjecture under stronger geometric assumptions.

Corollary 4.15 Let $M^{n}$ be a simply connected closed Riemannian manifold with one of the following:

- 2-positive curvature operator.

- Weakly quarter-pinched curvature.

- $\quad \sec \geq 1$ and $\operatorname{diam} M \geq \pi / 2$.

In each of these cases, $M$ satisfies the Wilhelm conjecture.

Proof In all three cases the respective results in $[5 ; 7 ; 15 ; 43]$ imply that $M$ is a compact rank-one symmetric space and therefore has singly generated cohomology.

Remark 4.16 A classical example of the type of fibration we are dealing with in Case 1 of the proof of Theorem 4.13 is the twistor bundle $\mathbb{S}^{2} \hookrightarrow \mathbb{C P}^{2 n+1} \rightarrow \mathbb{H P}^{n}$. An example of a non-trivial bundle in Case 2 is $\mathbb{S}^{3} \hookrightarrow \mathbb{S}^{4 n+3} \rightarrow \mathbb{H} \mathrm{P}^{n}$.

We proceed to the proof of Theorem A in the second case. It is contained in the following. 
Theorem 4.17 The Wilhelm conjecture holds true for all the known even-dimensional examples of manifolds admitting positive curvature.

Proof The CROSSes are covered by Theorem 4.13. According to [50] it remains to consider

(1) the flag manifolds $W^{6}=\mathrm{SU}(3) / T^{2}, W^{12}=\mathrm{Sp}(3) / \mathrm{Sp}(1)^{3}, W^{24}=\mathrm{F}_{4} / \operatorname{Spin}(8)$,

(2) $\mathrm{SU}(3) / / T^{2}$.

By [11, page 51] (and the formality of positively elliptic manifolds), the minimal model of $W^{6}$ is given by

$$
\left(\Lambda\langle a, b, x, y\rangle, a \mapsto 0, b \mapsto 0, x \mapsto a^{2}+a b+b^{2}, y \mapsto b^{3}\right)
$$

and that of $\mathrm{SU}(3) / / T^{2}$ by

$$
\left(\Lambda\langle a, b, x, y\rangle, a \mapsto 0, b \mapsto 0, x \mapsto a^{2}+a b-b^{2}, y \mapsto b^{3}\right)
$$

with $\operatorname{deg} a=\operatorname{deg} b=2$. The minimal model of $W^{12}$ is given by

$$
\left(\Lambda\langle a, b, x, y\rangle, a \mapsto 0, b \mapsto 0, x \mapsto a^{2}+a b+b^{2}, y \mapsto b^{3}\right)
$$

for $\operatorname{deg} a=\operatorname{deg} b=4$. The minimal model of $W^{24}$ can be derived from [29, Theorem 1.1 , where the integral cohomology algebra of $W^{24}$ is given. It computes as

$$
\left(\Lambda\langle a, b, x, y\rangle, a \mapsto 0, b \mapsto 0, x \mapsto a^{2}-a b+b^{2}, y \mapsto b^{3}\right)
$$

with $\operatorname{deg} a=\operatorname{deg} b=8$. (For this we denote the generators of the integral cohomology $\left(2 e\left(\mathcal{E}_{1}\right)+e\left(\mathcal{E}_{2}\right)\right) / 3$ by $a$ and $\left(e\left(\mathcal{E}_{1}\right)+2 e\left(\mathcal{E}_{2}\right)\right) / 3$ by $b$ and the relations are then given as the second and third elementary symmetric polynomials in $(a, b-a,-b)$, using the terminology from [29, Theorem 1.1].)

Let $E$ be a closed, simply connected manifold, rationally equivalent to one of these four spaces. Let $F \hookrightarrow E \rightarrow B$ be a non-trivial fibration of simply connected manifolds. We need to prove that $\operatorname{dim} F<\operatorname{dim} B$.

As in the proof of Theorem 4.13, both the fiber and the base are rationally elliptic of positive Euler characteristic. Thus the rational cohomology module of the total space splits as a product of the ones of fiber and base, since the Leray-Serre spectral sequence degenerates at the $E_{2}$-term for degree reasons. Consequently, the generators of the cohomology algebra of $B$ map injectively into the cohomology algebra of $E$.

Given the concrete minimal models, note the following key observation: the squaring map $H^{d}(E) \rightarrow H^{2 d}(E), z \mapsto z^{2}$, is injective, where $d \in\{2,4,8\}$ in the respective 
cases. It follows that the composition

$$
H^{d}(B) \stackrel{p^{*}}{\rightarrow} H^{d}(E) \rightarrow H^{2 d}(E)
$$

is injective. This composition equals the composition

$$
H^{d}(B) \rightarrow H^{2 d}(B) \stackrel{p^{*}}{\rightarrow} H^{2 d}(E),
$$

where the first map is the squaring map. Since $B \chi_{\mathbb{Q}} *$ and $F \chi_{\mathbb{Q}} *$, it follows that $H^{2 d}(B)$ is non-trivial, and hence that $\operatorname{dim}(B) \geq 2 d$. Since $\operatorname{dim}(E)=3 d$, it follows that $\operatorname{dim}(B)>\operatorname{dim}(F)$.

Corollary 4.18 Let $M^{n}$ be an even-dimensional, positively curved, simply connected, closed Riemannian manifold with isometry group satisfying $\operatorname{dim} \operatorname{Isom}\left(M^{n}\right) \geq 2 n-6$. Then $M$ satisfies the Wilhelm conjecture.

Proof This follows directly from the classification results in Wilking [45] and Wallach [41], which together imply that $M$ is diffeomorphic to a CROSS or one of the three Wallach manifolds. The Wilhelm conjecture holds for these manifolds by Theorems 4.13 and 4.17 , so this concludes the proof.

Finally, we prove the third conclusion of Theorem A. It is contained in the following.

Theorem 4.19 If $M^{2 n}$ is a positively curved manifold with the rational homotopy type of a simply connected hermitian symmetric space $N$, and if $M$ has symmetry rank at least $2 \log _{2}(2 n)+6$, then $N$ is either $\mathbb{C P}^{n}$ or $\mathrm{SO}(n+2) / \mathrm{SO}(2) \times \mathrm{SO}(n)$. Moreover, $M$ gives a positive answer to Question 4.2, unless $n$ is even, $N=\mathrm{SO}(n+2) / \mathrm{SO}(2) \times$ $\mathrm{SO}(n)$, and the fibration has fiber $F \simeq \mathbb{Q} \mathbb{C P}^{n / 2}$ and base $B \simeq \mathbb{Q} \mathbb{S}^{n}$.

Proof The classification result is Proposition 2.6. In the case of a rational $\mathbb{C P}^{n}$ we may quote Theorem 4.13. In the case of $\mathrm{SO}(n+2) / \mathrm{SO}(2) \times \mathrm{SO}(n)$, we argue as follows. The minimal model of $\mathrm{SO}(n+2) / \mathrm{SO}(2) \times \mathrm{SO}(n)$ is the one of $\mathbb{C P}^{n}$ if $n$ is odd, and is

$$
\left(\Lambda\left\langle u_{2}, a_{n}, x, y\right\rangle, x \mapsto a^{2}+u^{n}, y \mapsto a u\right)
$$

if $n$ is even. We may assume therefore that $n$ is even. Arguing as in the previous proofs, we have that $M, F$, and $B$ are $F_{0}$ spaces, and hence the spectral sequence degenerates at the $E_{2}$ term. In particular, $p^{*}: H^{*}(B) \rightarrow H^{*}(M)$ is injective.

We claim that either $\operatorname{dim} F<\operatorname{dim} B$, or $B \simeq \mathbb{Q}_{\mathbb{S}^{n}}$. First, if $H^{i}(B) \neq 0$ for some $i>n$, then $\operatorname{dim} B>n=\frac{1}{2} \operatorname{dim} M$, so $\operatorname{dim} F<\operatorname{dim} B$. Second, suppose there is a non-zero element $v \in H^{i}(B)$ with $0<i<n$. The pullback $p^{*}(v) \in H^{i}(M)$ is a non-zero 
multiple of $u^{j}$ for some $j<n / 2$. It follows by successive squaring that some power of $v$ is both non-zero and of degree larger than $n$. Hence $\operatorname{dim}(B)>n=\frac{1}{2} \operatorname{dim}(M)$ again. Since $B$ is a simply connected, closed, rationally $(n-1)$-connected manifold of dimension at most $n$, it is a rational $\mathbb{S}^{n}$. It follows now that $F \simeq \mathbb{Q} \mathbb{C P}^{n / 2}$ and $\operatorname{dim} F=\operatorname{dim} B$.

We remark that there is indeed a rational splitting of

$$
\left(\Lambda\langle u, a, x, y\rangle, x \mapsto a^{2}+u^{n}, y \mapsto a u\right)
$$

into a rational $\mathbb{C P}^{n / 2}$-fibration over $\mathbb{S}^{n}$ which, for example, is determined by

$$
\left(\Lambda\langle u, a, x, y\rangle, x \mapsto a^{2}, y \mapsto u^{n / 2+1}-a u\right)
$$

with $a, x$ corresponding to the base, $u, y$ to the fiber and with the isomorphism

$$
\left(\Lambda\langle u, a, x, y\rangle, x \mapsto a^{2}, y \mapsto u^{n / 2+1}-a u\right) \rightarrow\left(\Lambda\langle u, a, x, y\rangle, x \mapsto a^{2}+u^{n}, y \mapsto a u\right)
$$

determined by $u \mapsto u, a \mapsto a+u^{n / 2}$ and the formality of the morphism.

Theorem 4.19 completes the proof of Theorem A. In the rest of this section, we elaborate on the proof of the last case in Theorem 4.19. Hermitian symmetric spaces are examples of rationally elliptic manifolds that satisfy the Hard Lefschetz property. By considering this larger class of spaces, we generalize Theorem 4.19 as follows.

Proposition 4.20 Let $M^{n}$, for $n$ even, be hard Lefschetz, rationally elliptic, positively curved with symmetry rank at least $n / 8+2 \log _{2} n$. If $M$ fits into a fibration

$$
F \hookrightarrow M \stackrel{p}{\rightarrow} B
$$

as in Question 4.2, then $\operatorname{dim} F \leq \operatorname{dim} B+n / 2-2$, and $\operatorname{dim} F \geq \operatorname{dim} B$ only in the case of a rational $\mathbb{C P}^{(n-l) / 2}$-fibration over $\mathbb{S}^{l}$.

Proof This is the situation we are investigating in Corollary 2.4. Thus we derive that we are either in the case of a complex projective space - here we may quote Theorem 4.13 - or the minimal model of $M$ has the very special structure provided by the corollary.

As in Theorem 4.13 we may assume $F$ and $B$ to be rationally elliptic. First we argue that - unless the fibration is trivial — the hard Lefschetz form $[u]$ has to lie in the fiber cohomology; by this we refer to $[u] \in H^{*}(M)$ mapping to a non-trivial element in $H^{*}(F)$ under the projection $H^{*}(M) \rightarrow H^{*}(F)$. Indeed, if it comes from the base - ie if it lies in the image of $H^{*}(B) \rightarrow H^{*}(M)$ - we compute $0=[u]^{\operatorname{dim} B+1}=$ $\left(H^{2}(p)([u])\right)^{\operatorname{dim} B+1} \neq 0$ unless $\operatorname{dim} B=\operatorname{dim} M$. 
These considerations show that we have to differentiate between two cases. The cohomology class $[u]$ comes from the fiber, which implies for degree reasons that $u \in W$ where $(\Lambda W, \overline{\mathrm{d}})$ denotes the minimal model of the fiber $F$ and $(\Lambda V, \mathrm{~d})$ is the one of the base $B$. There is a second generator of the minimal model $(\Lambda(V \oplus W), \mathrm{d})$ in Corollary 2.4, namely $a \in V \oplus W$. We may assume that $a \in V$ or $a \in W$. For this, again we split

$$
(\Lambda(V \oplus W), \mathrm{d}) \cong\left(\Lambda V^{\prime}, \tilde{\mathrm{d}}\right) \otimes\left(\Lambda C, \widetilde{\mathrm{d}}_{0}\right)
$$

with $(\Lambda V, \mathrm{~d})$ a minimal model of $B$ and $(\Lambda W, \overline{\mathrm{d}})$ a minimal model of $F$, with a minimal Sullivan algebra $\left(\Lambda V^{\prime}, \tilde{\mathrm{d}}\right)$, and with a contractible algebra $\left(\Lambda C, \tilde{\mathrm{d}}_{0}\right)$ (see $[12$, Theorem 14.9, page 187]). We see that $a \in V^{\prime}$ by the uniqueness of minimal models. This implies that there has to exist a d-closed element in $V$ or a $\overline{\mathrm{d}}$-closed element in $W$, which, considered as an element of $(\Lambda(V \oplus W), \mathrm{d})$ is still closed and non-exact. We identify this element with $a$ and, depending on these two cases, we shall speak of $a$ as "coming from/lying in the base" respectively as "being from the fiber".

More precisely, in general, $\mathrm{d} a$, respectively $\overline{\mathrm{d}} a$, actually only has to lie in $\mathrm{d}\left(\Lambda^{\geq 2}(V \oplus\right.$ $W)$ ). However, since $\operatorname{deg} a$ is even, due to the arguments involving pure models of fibrations below, this need not be taken into consideration, since we may assume that $\mathrm{d} a=0$.

(Note that a sum of elements $v \in V$ and $w \in W$ with $\mathrm{d} v \neq 0$ and $\overline{\mathrm{d}} w \neq 0$ can never be closed in $(\Lambda(V \oplus W), \mathrm{d})$ (ie $\mathrm{d}(v+w) \neq 0)$, since $(\Lambda V, \mathrm{~d})$ is a differential subalgebra of $(\Lambda(V \oplus W), \mathrm{d})$, which implies that $\mathrm{d} w=-\mathrm{d} v \in \Lambda V$ and $\overline{\mathrm{d}} w=0$. Then $\overline{\mathrm{d}} w$ cannot be exact (neither with respect to $\overline{\mathrm{d}}$ nor to $\mathrm{d})$, since $(\Lambda W, \overline{\mathrm{d}})$ was chosen a minimal Sullivan algebra.)

Let us now consider these two cases separately: either $a$ comes from the fiber or the base.

Case 1 Assume both the elements $u$ and $a$ lie in the minimal model of the fiber. Let us show that in this case $\operatorname{dim} B=0$ and the fibration is trivial. For this we show that the model of the fibration is minimal already, ie the algebra $\left(C, \widetilde{\mathrm{d}}_{0}\right)$ from (8) is trivial; clearly, a stronger statement than just confirming Question 4.2 in this case.

Since $M$ has positive Euler characteristic, again we derive $\chi(F)>0, \chi(B)>0$. This implies that $B$ and $F$ are positively elliptic and the Leray-Serre spectral sequence degenerates at the $E_{2}$-term. Again, we may assume that the model of the fibration is

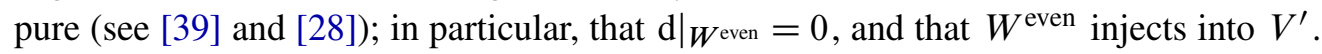
Together with the assumption that both $u$ and $a$ come from the fiber, this proves that

$$
2=\operatorname{dim}\left(V^{\prime}\right)^{\text {even }}=\operatorname{dim} W^{\text {even }}=\operatorname{dim} W^{\text {odd }} .
$$


The differential d may only have a non-trivial linear part on $W^{\text {odd }}$. Thus $\operatorname{dim} C^{\text {even }} \leq 2$. Consequently, $(\Lambda V, \mathrm{~d})$ satisfies $\operatorname{dim} V^{\text {even }} \leq 2$.

However, it is now easy to check that one cannot specify elements $x^{\prime}, y^{\prime} \in W^{\text {odd }}$ (together with a suited minimal model of the base) with $\overline{\mathrm{d}} x^{\prime} \neq 0, \overline{\mathrm{d}} y^{\prime} \neq 0$ and $\left.\left(\mathrm{d} x^{\prime}\right)\right|_{V} \neq$ $0,\left.\left(\mathrm{~d} y^{\prime}\right)\right|_{V} \neq 0$ such that the given minimal model of $M$ can result. This is due to the fact that $a^{2}$ and $a u^{(n-2 \operatorname{deg} a+2) / 2}$ are summands of the respective differentials in $\left(\Lambda V^{\prime}, \tilde{\mathrm{d}}\right)$. That is, both differentials contain the factor $a$ in a summand, however, since $a$ appears in its second power at most, the given relations cannot come from relations of the base (after gluing). The same holds true if there is exactly one generator in even degree in $C$. Thus $C$ has to be trivial.

Case 2 Assume $u$ is in the minimal model of the fiber and $a$ lies in the minimal model of the base. Then the element $x$ (from the minimal model specified in Corollary 2.4) must lie in the minimal model of the base, otherwise the base could not be finitedimensional. (More precisely, there must exist an element whose differential has a power of $a$ as a summand; for degree reasons we then see that this must correspond to $x$.)

The contractible algebra $\Lambda C$ contains at most one generator in $C^{\text {even }}$. As in Case 1 this follows since both $F$ and $B$ are positively elliptic, the spectral sequence degenerates at the $E_{2}$-term, the model of the fibration may be assumed to be pure and, consequently, $1=\operatorname{dim} W^{\text {even }}=\operatorname{dim} W^{\text {odd }}$; the linear part of $\mathrm{d}$ may only be non-trivial on $W^{\text {odd }}$ and $C^{\text {even }} \leq 1$.

So we differentiate these two cases, either $C^{\text {even }}=1$ or $C$ is trivial. A direct check shows that this corresponds to exactly two different cases on the level of models. Either the model of the fibration is given by

$$
\begin{aligned}
\left(\Lambda\left\langle u, x^{\prime}, u^{\prime}, x, a, y\right\rangle, \quad x^{\prime}\right. & \mapsto u^{\prime}, \quad u \mapsto 0, \quad u^{\prime} \mapsto 0, \\
x & \mapsto a^{2}+k_{1} a\left(u^{\prime}\right)^{\operatorname{deg} a / \operatorname{deg} u^{\prime}}+k_{2} \cdot\left(u^{\prime}\right)^{2 \operatorname{deg} a / \operatorname{deg} u^{\prime}}, \\
y & \left.\mapsto a\left(u^{\prime}\right)^{(n-2 \operatorname{deg} a+2) / \operatorname{deg} u^{\prime}}+k_{3}\left(u^{\prime}\right)^{(n-\operatorname{deg} a+2) / \operatorname{deg} u^{\prime}}\right)
\end{aligned}
$$

or by

$$
\left(\Lambda\langle u, x, a, y\rangle, \quad x \mapsto a^{2}, \quad y \mapsto u^{(n-\operatorname{deg} a+2) / 2}+k \cdot a u^{(n-2 \operatorname{deg} a+2) / 2}\right)
$$

with $k \neq 0$ in the second case. In the first case the model of the base is generated by $u^{\prime}, a, x, y$ and the one of the fiber by $u, x^{\prime}$. Consequently, we have that $\operatorname{dim} B>$ $\operatorname{dim} F$. In the second case the model is minimal already. The model of the base is generated by $a, x$ and the model of the fiber by $u, y$. This case corresponds to a (rational) $\mathbb{C P}^{(n-\operatorname{deg} a) / 2}$-fibration over $\mathbb{S}^{\operatorname{deg} a}$. Since $\operatorname{deg} a \geq n / 4+1$, we compute $\operatorname{dim} F-\operatorname{dim} B=(n-\operatorname{deg} a)-\operatorname{deg} a \leq n-2(n / 4+1)=n / 2-2$. 


\section{References}

[1] C Allday, V Puppe, Cohomological methods in transformation groups, Cambridge Studies in Advanced Mathematics 32, Cambridge Univ. Press (1993) MR1236839

[2] M Amann, Positive quaternion Kähler manifolds, $\mathrm{PhD}$ thesis, Westfälische WilhelmsUniversität Münster (2009) Available at http://d-nb.info/996176438/34

[3] M Amann, L Kennard, Topological properties of positively curved manifolds with symmetry, Geom. Funct. Anal. 24 (2014) 1377-1405 MR3261629

[4] I Belegradek, V Kapovitch, Obstructions to nonnegative curvature and rational homotopy theory, J. Amer. Math. Soc. 16 (2003) 259-284 MR1949160

[5] M Berger, Sur quelques variétés riemanniennes suffisamment pincées, Bull. Soc. Math. France 88 (1960) 57-71 MR0133781

[6] A Blanchard, Sur les variétés analytiques complexes, Ann. Sci. Ecole Norm. Sup. 73 (1956) 157-202 MR0087184

[7] C Böhm, B Wilking, Manifolds with positive curvature operators are space forms, Ann. of Math. 167 (2008) 1079-1097 MR2415394

[8] W Browder, Fiberings of spheres and H-spaces which are rational homology spheres, Bull. Amer. Math. Soc. 68 (1962) 202-203 MR0148071

[9] X Chen, Curvature and Riemannian submersions, $\mathrm{PhD}$ thesis, University of Notre Dame (2014) Available at http://search.proquest.com/docview/1547351162

[10] O Dearricott, A 7-manifold with positive curvature, Duke Math. J. 158 (2011) 307-346 MR2805071

[11] J-H Eschenburg, Freie isometrische Aktionen auf kompakten Lie-Gruppen mit positiv gekrümmten Orbiträumen, Schriftenr. Math. Inst. Univ. Münster (II) 32, Mathematisches Institut, Universität Münster (1984) MR758252

[12] Y Félix, S Halperin, J-C Thomas, Rational homotopy theory, Graduate Texts in Mathematics 205, Springer, New York (2001) MR1802847

[13] Y Félix, J Oprea, D Tanré, Algebraic models in geometry, Oxford Graduate Texts in Mathematics 17, Oxford Univ. Press (2008) MR2403898

[14] L A Florit, W Ziller, Topological obstructions to fatness, Geom. Topol. 15 (2011) 891-925 MR2821566

[15] D Gromoll, K Grove, A generalization of Berger's rigidity theorem for positively curved manifolds, Ann. Sci. École Norm. Sup. 20 (1987) 227-239 MR911756

[16] K Grove, Geometry of, and via, symmetries, from: "Conformal, Riemannian and Lagrangian geometry", (A Freire, editor), Univ. Lecture Ser. 27, Amer. Math. Soc. (2002) 31-53 MR1922721 
[17] K Grove, Developments around positive sectional curvature, from: "Geometry, analysis, and algebraic geometry: forty years of the Journal of Differential Geometry", (H-D Cao, S-T Yau, editors), Surv. Differ. Geom. 13, Int. Press, Somerville, MA (2009) 117-133 MR2537084

[18] K Grove, S Halperin, Dupin hypersurfaces, group actions and the double mapping cylinder, J. Differential Geom. 26 (1987) 429-459 MR910016

[19] K Grove, C Searle, Positively curved manifolds with maximal symmetry-rank, J. Pure Appl. Algebra 91 (1994) 137-142 MR1255926

[20] K Grove, L Verdiani, W Ziller, An exotic $T_{1} \mathbb{S}^{4}$ with positive curvature, Geom. Funct. Anal. 21 (2011) 499-524 MR2810857

[21] S Halperin, Rational fibrations, minimal models, and fibrings of homogeneous spaces, Trans. Amer. Math. Soc. 244 (1978) 199-224 MR0515558

[22] S Halperin, Polynomial growth and elliptic spaces, unpublished (2012)

[23] IM James (editor), Handbook of algebraic topology, North-Holland, Amsterdam (1995) MR1361885

[24] L Kennard, On the Hopf conjecture with symmetry, Geom. Topol. 17 (2013) 563-593 MR3039770

[25] L Kennard, Positively curved Riemannian metrics with logarithmic symmetry rank bounds, Comment. Math. Helv. 89 (2014) 937-962 MR3284301

[26] M Kerin, Some new examples with almost positive curvature, Geom. Topol. 15 (2011) 217-260 MR2776844

[27] G Lupton, Note on a conjecture of Stephen Halperin's, from: "Topology and combinatorial group theory”, (P Latiolais, editor), Lecture Notes in Math. 1440, Springer, Berlin (1990) 148-163 MR1082989

[28] G Lupton, Variations on a conjecture of Halperin, from: "Homotopy and geometry", (J Oprea, A Tralle, editors), Banach Center Publ. 45, Polish Acad. Sci., Warsaw (1998) 115-135 MR1679854

[29] A-L Mare, M Willems, Topology of the octonionic flag manifold, Münster J. Math. 6 (2013) 483-523 MR3148220

[30] M Markl, Towards one conjecture on collapsing of the Serre spectral sequence, from: "Proceedings of the Winter School on Geometry and Physics", (J Bureš, V Souček, editors), Rend. Circ. Mat. Palermo Suppl. 22 (1990) 151-159 MR1061796

[31] W Meier, Rational universal fibrations and flag manifolds, Math. Ann. 258 (1982) 329-340 MR649203

[32] W Meier, Some topological properties of Kähler manifolds and homogeneous spaces, Math. Z. 183 (1983) 473-481 MR710764 
[33] S Papadima, L Paunescu, Reduced weighted complete intersection and derivations, J. Algebra 183 (1996) 595-604 MR1399041

[34] P Petersen, F Wilhem, An exotic sphere with positive curvature, preprint (2008) arXiv:0805.0812v3

[35] T Püttmann, C Searle, The Hopf conjecture for manifolds with low cohomogeneity or high symmetry rank, Proc. Amer. Math. Soc. 130 (2002) 163-166 MR1855634

[36] X Rong, X Su, The Hopf conjecture for manifolds with abelian group actions, Commun. Contemp. Math. 7 (2005) 121-136 MR2129791

[37] H Shiga, M Tezuka, Rational fibrations, homogeneous spaces with positive Euler characteristics and Jacobians, Ann. Inst. Fourier (Grenoble) 37 (1987) 81-106 MR894562

[38] Z Su, Rational analogs of projective planes, Algebr. Geom. Topol. 14 (2014) 421-438 MR3158765

[39] J-C Thomas, Rational homotopy of Serre fibrations, Ann. Inst. Fourier (Grenoble) 31 (1981) v, 71-90 MR638617

[40] J M Wahl, Derivations, automorphisms and deformations of quasihomogeneous singularities, from: "Singularities, Part 2", (P Orlik, editor), Proc. Sympos. Pure Math. 40, Amer. Math. Soc. (1983) 613-624 MR713285

[41] N R Wallach, Compact homogeneous Riemannian manifolds with strictly positive curvature, Ann. of Math. 96 (1972) 277-295 MR0307122

[42] G Walschap, Soul-preserving submersions, Michigan Math. J. 41 (1994) 609-617 MR1297712

[43] B Wilking, Index parity of closed geodesics and rigidity of Hopf fibrations, Invent. Math. 144 (2001) 281-295 MR1826371

[44] B Wilking, Torus actions on manifolds of positive sectional curvature, Acta Math. 191 (2003) 259-297 MR2051400

[45] B Wilking, Positively curved manifolds with symmetry, Ann. of Math. 163 (2006) 607-668 MR2199227

[46] B Wilking, Nonnegatively and positively curved manifolds, from: "Metric and comparison geometry", (J Cheeger, K Grove, editors), Surv. Differ. Geom. 11, Int. Press, Somerville, MA (2007) 25-62 MR2408263

[47] J E Yeager, Geometric and topological ellipticity in cohomogeneity two, PhD thesis, University of Maryland (2012) Available at http://hdl .handle.net/1903/12662

[48] O Zariski, P Samuel, Commutative algebra, I, Graduate Texts in Mathematics 28, Springer, New York (1975) MR0384768

[49] O Zariski, P Samuel, Commutative algebra, II, Graduate Texts in Mathematics 29, Springer, New York (1976) MR0389876 
[50] W Ziller, Examples of Riemannian manifolds with non-negative sectional curvature, from: "Metric and comparison geometry", (J Cheeger, K Grove, editors), Surv. Differ. Geom. 11, Int. Press, Somerville, MA (2007) 63-102 MR2408264

[51] W Ziller, Riemannian manifolds with positive sectional curvature, from: "Geometry of manifolds with non-negative sectional curvature", (R Herrera, L Hernández-Lamoneda, editors), Springer, Berlin (2014) 1-19

Fakultät für Mathematik, Institut für Algebra und Geometrie

Karlsruher Institut für Technologie, Englerstraße 2, D-76131 Karlsruhe, Germany

Department of Mathematics, University of Oklahoma

Norman, OK 73019, USA

manuel.amann@kit.edu, kennard@math.ou.edu

http://www.math.kit.edu/iag7/ amann

Received: 9 June $2014 \quad$ Revised: 22 October 2014 
